\title{
¿LA NEUROCIENCIA EDUCACIONAL COMO ESPACIO PROPIO DE LA EDUCACIÓN ES POSIBLE?
}

\author{
IS EDUCATIONAL NEUROSCIENCE POSSIBLE \\ AS A SPACE OF EDUCATION ITSELF?
}

\author{
Dra. María de los Ángeles Bacigalupe
}

\section{Resumen}

En este trabajo proponemos discutir la neurociencia educacional como espacio de la educación. Mediante el análisis y discusión bibliográfica indagamos las posibilidades de la neurociencia educacional en el contexto de su origen y actualmente, y avanzamos hacia los desafíos a los que se enfrenta como disciplina propia del campo de la educación o como rama de la neurociencia cognitiva. Optamos por una mirada transdisciplinaria que desde la propia educación pueda colaborar en los temas que preocupan a la educación como institución social. Proponemos una mirada que incluya tanto al nivel individual como el nivel socialmente extendido, considerando que el aprendizaje, como comportamiento humano que constituye un problema educacional, se da en interacción con el medio. Planteamos cuestiones presentes en la agenda interdisciplinaria de la neurociencia educacional y discutimos conceptos clave como neuromito, epigenética, privación psicosocial y periodo crítico. Proponemos que, concebida como espacio educacional transdisciplinario con funciones de traslación y ciencia básica, la neurociencia educacional, en diálogo con otras disciplinas, puede conducir a que la diversidad no sea un concepto teórico sino que se refleje en cada ser humano, en su potencialidad y originalidad, buscando el desarrollo pleno de las personas en relación armoniosa con su medioambiente de vida.

Palabras clave: Neurociencia educacional; Aprendizaje; Educación. 


\section{Abstract}

In the present article we propose to discuss educational neuroscience as a segment of education. We have analysed educational neuroscience from its origins to the present, as a branch of education sciences. We have chosen a transdisciplinary approach which views learning from an individual to a social perspective. In it, we have discussed key concepts such as neuromyth, epigenetics, psychological deprivation and critic period.

Key words: Educational neuroscience; Learning; Education.

\section{Introducción: Problema}

Los problemas que se presentan en el escenario educacional son altamente complejos, diversos, multicausales y multidimensionales. La complejidad de las sociedades del siglo XXI hace de este escenario un espacio desafiante para los educadores. Sin embargo, la formación docente no parece acompañar esos diagnósticos, a juzgar por los resultados que encontramos cotidianamente en fuentes de circulación pública, como tampoco parece hacerlo la misma institución educativa, como institución político-social con fines y funciones que en su momento fueron claras y precisas pero que en los últimos tiempos se han diversificado y a donde muchas cuestiones vinculadas a la educación asistemática y a la familia y crianza, como primera educación, han sido delegadas.

Por otro lado, esta misma complejización, diversificación y cambio ha atravesado al sistema científico y tecnológico, generando productos que pocos años atrás resultaban impensados, lo cual también trae aparejado un debate ético-social -del que no nos ocuparemos en este artículo.

Sería necio para cualquier área del sistema social no hacer uso de esta disponibilidad de conocimiento científico y de tecnología, tanto si hablamos del subsistema político como del económico, del sanitario y, obviamente, del educacional.

Sin embargo, una cuestión es el uso y otra el abuso, así como también es importante considerar que utilizar productos de un sistema para fines de otro implica un trabajo translacional que no significa aplicar simplemente sino que requiere de un trabajo especializado, tanto en la selección de productos (sean estos conocimientos, tecnología u otros) como en su adaptación activa y modificación pertinente para un uso apropiado en el subsistema que acoge la innovación, producto o servicio. 
Importante es destacar que lo nuevo no borrará de llano lo anterior sino que el proceso de adaptación requiere incorporación y flexibilidad de acomodación, así como que la idea no es que un subsistema receptor sea reemplazado por otro innovador.

En la relación que nos ocupa en este artículo, intersección entre educación y neurociencias, esto significa decir que nunca las neurociencias podrían reemplazar a la educación ni que los conocimientos de las neurociencias, por deslumbrantes que sean, puedan reemplazar a los conocimientos pedagógicos. No se trata de ser tan devotos de la innovación que caigamos en el aplicacionismo, ni tan críticos negacionistas que caigamos en la crítica destructiva y el dogmatismo de la crítica.

A todo esto, nos interesa aclarar el término neurociencias. En un texto bastante reciente, Ibáñez y García (2015) señalan que "las neurociencias son una empresa científica que explora la organización y las funciones del cerebro" (p. 22) y ya hacia el final de la obra retoman el tema diciendo que:

Estas pueden concebirse como un conjunto de subdisciplinas especializadas, cada una de las cuales se ocupa de un aspecto particular de la cognición (...) $\mathrm{Si}$ bien poseen intereses diversos, sus practicantes comparten el presupuesto de que el cerebro es el motor de la cognición (Ibáñez y García, 2015, p. 174)

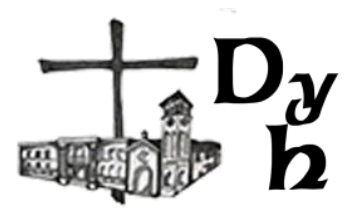

Las neurociencias abordan cuestiones centrales a la humanidad, tales como el desarrollo, el aprendizaje de habilidades culturales complejas como la lectura, el comportamiento y sus motores y la toma de decisiones y su interrelación con las emociones, cubriendo múltiples niveles de análisis (Ansari, König, Leask y Tokuhama-Espinosa, 2017).

La pregunta, entonces, es cómo pueden conectarse ambas áreas del saber. En la teoría la cuestión es bastante obvia, nos educamos con un cuerpo que está vivo y aprendemos con ese cuerpo en relación con un medioambiente. Tanto el cuerpo como el medio contienen o son materia viva y las neurociencias incluyen disciplinas que específicamente se ocupan de estudiar la materia viva en sus formas más diversas, su origen y evolución. El ser humano tiene una historia como especie Homo sapiens que se arraiga en toda la historia natural de la vida $y$, ontogenéticamente hablando, el individuo humano es tan biológicamente inmaduro cuando nace que si no fuera por su medio social no sobreviviría, su cuerpo, su biología no podría desarrollarse. Esa crianza inicial es la primera educación y esa inmadurez neurobiológica es la oportunidad de educarse: fundamenta la educabilidad de la especie. Pero en la práctica, la cuestión de relacionar neurociencia y educación es muy complicada y la opción no es que los neurocientíficos vengan a dar cursos a los educadores sobre sistema 
nervioso sino que lo que hace falta es crear un espacio educacional original y propio que se ocupe de este trabajo traslacional. Ese espacio es el de la neurociencia educacional.

\section{Qué es la neurociencia educacional}

La neurociencia educacional es una joven área de conocimiento inaugurada 20 años atrás a partir del artículo seminal de Bruer (1997) (Howard-Jones et al., 2016).

El conocimiento del cerebro y los avances de las neurociencias al respecto constituyen un atrayente campo cuya promoción se realiza actualmente por los medios de comunicación accesibles al público en general y que cuenta con divulgadores que intentan traducir, con más o menos éxito, el lenguaje científico al lenguaje cotidiano. Los educadores no se alejan de esta tendencia de atracción al conocimiento del cerebro. Sin embargo, que haya interés por el conocimiento del cerebro y que haya lectura sobre el tema no significa que se logre un aprendizaje que respete el conocimiento producido en los laboratorios de ciencia. De hecho, en la investigación de Dekker, Lee, Howard-Jones y Jolles (2012) se halló que los educadores con interés en el estudio del cerebro presentaban importantes concepciones equivocadas al respecto, o neuromitos.

La palabra neuromito se refiere a concepciones equivocadas acerca de los conocimientos de las ciencias del cerebro debido a una lectura errónea de esas investigaciones. Por ejemplo, cuenta entre los tradicionales neuromitos aquel que señala que usamos solamente el $10 \%$ del cerebro (Dündar y Gündüz, 2016).

La clara existencia de neuromitos en los responsables de la educación formal constituye un motivo suficiente para despejar prejuicios y la única forma de hacerlo es enseñando ciencia, que es lo contrario al prejuicio y al dogma, lo cual es una de las razones para la construcción de una neurociencia educacional.

Por otro lado, si la educación pudiera resolver sus problemas y viéramos cambios positivos para la población, no haría falta la discusión sobre si las neurociencias pueden aportar algo a la educación. Sin embargo, tal como señala Gabrieli (2016) y muchos otros autores y así como muestran los diarios $y$ periódicos de circulación general y las noticias por redes virtuales $y$ demás, la educación está lejos de alcanzar los resultados que la sociedad demanda y los más perjudicados son los sectores más vulnerables.

Varios autores identifican a la neurociencia educacional como rama o área de la neurociencia cognitiva (Bowers, 2016; Gabrieli, 2016; Lipina, 2016) cuyo métier es realizar el trabajo de traslación desde la investigación en neurociencia básica a la práctica educativa (Gabrieli, 2016). 
Desde las ciencias de la educación, nosotros ya hablábamos de la interdisciplina entre educación y neurociencias en la publicación de 2001 en una revista británica de profesores de idioma inglés (Bacigalupe, 2001). Sin embargo, esta perspectiva priorizaba que la nueva interdisciplina surgía como un área conformada desde y en el gran campo de estudios educacionales.

En su artículo de 1997, Bruer señalaba que querer establecer la relación entre neurociencias y educación era como querer construir un puente con instancias muy alejadas una de otra (a bridge too far) pero que había dos puentes más cortos que indirectamente podían acercar la relación entre el cerebro y la educación: el primero, entre educación y psicología cognitiva y, el segundo, entre psicología cognitiva y neurociencias (Bruer, 1997). Su negativa a relacionar directamente neurociencias con educación se basaba en algunas cuestiones relevantes, tales como la forma incorrecta desde la educación de leer los resultados de las neurociencias y el conocimiento limitado que ofrecían las neurociencias sobre el cerebro humano -y más limitado aún sobre el cerebro humano en relación a las habilidades tradicionalmente escolares.

Casi 20 años después, el mismo autor (Bruer, 2016a) se ha ocupado de aclarar que lo que él llamaba neurociencia en aquél momento era la neurofisiología del desarrollo, basada en los conceptos de sinaptogénesis, periodos críticos y enriquecimiento del ambiente que era popular entre los educadores y defensores de la neurociencia en el aula de aquellos años, es decir que no se refería a la totalidad de neurociencias. Con su mirada en el año 2016, el autor ve a la neurociencia educacional como neurociencia cognitiva educacional.

Bowers (2016s) en su crítica a la neurociencia educacional señala que esta disciplina no ha traído avances concretos al diseño de prácticas educacionales en el aula y que no agrega cuestiones nuevas a las que la psicología no ha aportado ya a la educación, señalando que la psicología es suficiente para informar a la educación (Bowers, 2016b).

Podría argumentarse que esta concepción de educación queda restringida a la educación formal, cuando la educación incluye también los ámbitos no formal e informal e implica la formación del ser humano desde el nacimiento hasta el deceso.

Por otro lado, la concepción de neurociencia que aparece en el argumento de Bowers es una concepción restringida, distinta a la idea de neurociencias plural e interdisciplinaria que incluye tanto a la psicología y la economía como a la antropología (cfr. Ansari et al., 2017), por nombrar disciplinas clave que aportan elementos esenciales a la comprensión de la cognición incorporada y culturalmente adaptada que se sostiene en códigos neurobiológicos comportamentales. Una visión 
amplia de las neurociencias con conexiones fuertes con la psicología y la antropología ya aparecía en la conocida obra de Howard Gardner The mind's new science de 1985 (Gardner, 1987).

Gabrieli (2016) señala que "La neurociencia educacional puede ser especialmente pertinente para la cantidad de niños con diferencias cerebrales que hacen que el progreso educativo sea difícil en el curriculum estándar" (Gabrieli, 2016, p. 614$)^{1}$.

Acordamos con el autor en que la neurociencia educacional puede aportar elementos útiles para estas cuestiones, pero no consideramos que sea su último objetivo ser una disciplina que en su momento se hubiera llamado educación especial. Cabe señalar que el autor indica que los mejores resultados de la neurociencia educacional al momento se refieren a estudiantes con dificultades de aprendizaje y que no está claro cómo podrá realizar su labor traslacional para la población completa de estudiantes, aventurando que quizás lo logre proveyendo elementos para un curriculum individualizado, que pueda respetar y apoyar las diferencias entre las personas.

\footnotetext{
1 "Educational neuroscience may be especially pertinent for the many children with brain differences that make educational progress difficult in the standard curriculum" (Gabrieli, 2016, p. 614, esta y las siguientes traducciones del original en el texto son de la autora)
}

Conocer las diferencias individuales sería una labor predictiva de la neurociencia educacional que permitiría, por ejemplo, que la educación se ocupara de eliminar los círculos viciosos de fracaso tras fracaso en los que se ven envueltos algunos estudiantes (Gabrieli, 2016), no por tener déficits cognitivos sino por tener habilidades para desarrollarse mejor en unos ambientes más que en otros. Sostenemos que esta perspectiva resulta necesaria ya que los estudiantes desfavorecidos están en condiciones inferiores que el resto de los estudiantes simplemente porque la educación no ha logrado encontrar la forma de que aprendan, lo cual es un problema de la educación, no del estudiante como individuo.

Conocer las diferencias individuales permitiría también establecer integraciones en el aula que fueran capaces de favorecer a todo el grupo de clase involucrado, incluyendo a los docentes responsables y a la institución educacional, comprendiendo lo que significa la integración y evitando la generación de conflictos. Al fin y al cabo, todos somos en alguna medida integrados y en conjunto formamos un grupo, todos somos distintos y el grupo es el resultado de la labor de integración que nos favorece a todos, donde todos damos y recibimos. Esto tiene que ver no sólo con la integración de niños con capacidades diferentes en el aula de clase común, sino con la integración de niños inmigrantes, por ejemplo. 
Reconocemos que todos somos diferentes y que gracias a la diferencia $y$ al intercambio de diferentes personas es que nuestra especie ha sobrevivido, entre otras cuestiones, ya que un principio básico de la evolución es la diversidad (puede revisarse una mirada de la teoría evolucionista en Mayr, 1998; una excelente exposición de la historia y perspectiva actual de la teoría puede verse en Magazine de Ciencia, 2017).

En el nombre neurociencia educacional, la palabra neurociencia significa neurociencia cognitiva, según Howard-Jones et al. (2016), cuya función es establecer vínculos entre el sustrato neural de los procesos mentales y los comportamientos, especialmente el comportamiento referido al aprendizaje que ocurre en la educación formal.

En su defensa sobre el sentido de ser de la neurociencia educacional, Gabrieli (2016) discute la postura de Bowers (2016) y señala que hay buenas razones para esperar que la neurociencia educacional provea resultados que sean relevantes para la educación. Sin embargo, esto no significa, según el autor, pensar que la neurociencia educacional traerá soluciones directamente aplicables al aula, ya que es per se una ciencia básica.

Hay algunos avances, que el autor cita, señalando las posibilidades de esta disciplina, concluyendo:

Parafraseando a George Bernard Shaw, creo que Bowers ve las limitaciones de la neurociencia educacional y pregunta "Por qué", y yo veo la cantidad de necesidades de los niños y la evidencia nueva y prometedora que la neurociencia educacional está aportando acerca de las diferencias cerebrales que puede transformar la educación individualizada, y digo "¿Por qué no?" (Gabrieli, 2016, p. 617)2.

\section{Aprendizaje}

Una cuestión a abordar es la diferencia epistemológica entre neurociencias y educación, comenzando por qué entiende cada una por aprendizaje, asumiendo que la neurociencia educacional tiene el objetivo de "combinar nuestra comprensión educacional con nuestra comprensión biológica de la función cerebral y el aprendizaje" (Howard-Jones, 2008, p. 361) . $^{3}$.

El término aprendizaje no significa lo mismo para la educación y para las neurociencias. En neurociencias el aprendizaje se entiende en términos de formación

\footnotetext{
2 To paraphrase George Bernard Shaw, I believe that Bowers sees the limitations of educational neuroscience and asks "Why," and I see the many needs of children and the promising and novel evidence that educational neuroscience is providing about brain differences that can transform individualized education, and say "Why not?" (Gabrieli, 2016, p. 617)

3 "to combine our educational
understanding with our biological
understanding of brain function and
learning" (Howard-Jones, 2008, p. 361)
}


de memoria, como un fenómeno que ocurre mediante cambios en los patrones de conectividad entre neuronas, llamado plasticidad sináptica, del cual se han probado al menos dos tipos (in vivo con animales no humanos e in vitro), la potenciación de largo plazo o longterm potentiation (LTP - un aumento duradero de la eficiencia con que una neurona transmite señales eléctricas a otra gracias al emparejamiento temporal entre las señales de entrada y de salida, cfr. Lømo, 2003) y la depresión de largo plazo o long-term depression (LTD una disminución duradera de la eficacia sináptica, cfr. Ito, 2001). Esta hipótesis del aprendizaje basado en las formas de plasticidad sináptica convive con la hipótesis genómica en la cual las modificaciones al ADN sirven como portadores de trazos de memoria. Asimismo se habla de tipos de actividad cerebral donde lo que prevalece es la reactivación de memorias almacenadas, lo cual se relaciona con el fenómeno de la memoria de trabajo, tan importante en la educación de las personas porque contiene mecanismos necesarios para la resolución de problemas. Otra forma de modificación asociada al aprendizaje distinta de la sináptica es el cambio estructural en el cerebro.

Desde las neurociencias moleculares llegan también otras nociones que hablan del aprendizaje como relación individuo-entorno, donde destaca el estudio de la epigenética, es decir, los cambios que ocurren en el ADN y que regulan la expresión génica, tales como metilación y acetilación, como resultado de la interrelación entre el organismo y su ambiente. Un atención especial merecen para la neurociencia educacional los estudios de epigenética que asocian los mecanismos moleculares de expresión génica con los conceptos de memoria y aprendizaje (cfr. por ejemplo Blaze y Roth, 2013).

Frente a esta conceptualización neurobiológica, en educación la concepción de aprendizaje es variable y ecléctica (Howard-Jones, 2008). Se vislumbran en la misma rasgos constructivistas con la idea de una práctica escolar que valore el conocimiento previo y promueva el aprendizaje independiente, que considere el aprendizaje en equipo y en contexto, el aprendizaje informal, la formación continua del docente y su rol de andamiaje en el proceso del aprendiz.

Uno de los problemas que surgen al querer concatenar el concepto educacional de aprendizaje con el neurocientífico es que se puede caer en un monismo sin sentido (tal como creer que los estados mentales pueden ser descriptos completamente en términos cerebrales y que existe una evidencia biológica conclusiva para tener una idea psicológica de cómo funciona la mente) o un dualismo (tal como entender la mente como un concepto teórico sustancialmente distinto a los procesos neurobiológicos). Howard-Jones 
(2008) sugiere una mirada diferente. A partir de la noción de la neurociencia cognitiva que considera que cerebro y mente pueden explicarse en conjunto (la mente constituye un concepto teórico esencial en la indagación de las relaciones entre cerebro y comportamiento, incluyendo el aprendizaje), el autor señala una vía para reconciliar concepciones entre neurociencias y educación. En el modelo de la neurociencia cognitiva se conjugan distintos niveles de descripción, comportamiento, procesos biológicos, cognición (como subnivel entre los niveles biológico y comportamental) y medioambiente (influenciando los otros niveles e incluyendo desde nutrientes y oxígeno hasta herramientas de enseñanza). En este modelo el enlace causal suele buscarse usualmente desde cerebro a mente y de allí al comportamiento, incluyendo, por supuesto, la complejidad del establecimiento de causas. Sin embargo, la influencia puede ser bidireccional y esta bidireccionalidad, de gran interés para los educadores, incluye un elemento no menor en el modelo anteriormente descripto que lo hace más apropiado como arquetipo de la neurociencia propiamente educacional. Asimismo, y tomando como base el modelo basado en el nivel individual de la neurociencia cognitiva, la neurociencia educacional propone un modelo socialmente extendido (HowardJones, 2008), integrando aportes tanto de las ciencias naturales como de las ciencias sociales (no dejando afuera ni el nivel de descripción neurobiológica ni el estudio de los significados de las acciones en las relaciones humanas).

\section{Interdisciplinariedad en educación: cuestiones de agenda}

Uno de los argumentos centrales que utiliza Gabrieli (2016) para defender el espacio de la neurociencia educacional como disciplina que tenga alguna incidencia en la educación es la dificultad en que se encuentra la educación para resolver sus problemas, que no ha podido salvar en tantos años, y la posibilidad de interdisciplinariedad entre las ciencias del comportamiento y las ciencias del cerebro -contando entre otros argumentos el hecho de que el órgano del comportamiento es efectivamente el cerebro.

Lejos de identificarnos con la postura completa de este autor, sí acordamos en su defensa de la potencialidad que puede tener la combinación de metodologías y teorías en educación.

Howard-Jones et al (2016) sostienen que los niveles de explicación psicológico y neural se complementan mutuamente. La ruta entre neurociencias y educación es compleja e indirecta, sostienen los autores, y si bien la eficacia de una intervención educacional se mide mediante métodos comportamentales, en intervenciones educacionales que han sido 
informadas tanto por datos comportamentales como neurales la evaluación mediante métodos neurobiológicos puede ser importante para entender cómo se está logrando la eficacia de la intervención, conservando la medida de la eficacia de la intervención per se mediante métodos comportamentales. De este modo se logra una complementación de información (datos recolectados en el aula de clases y datos provenientes de la investigación neurocognitiva) donde se ven beneficiadas ambas disciplinas intervinientes (Howard-Jones et al, 2016).

Por otro lado, en esta interdisciplina no hay dualismo o jerarquía de disciplinas sino, por el contrario, una complementación que incluye distintos niveles de explicación que juntos sirven para mejorar el conocimiento que tenemos del desarrollo y aprendizaje (Howard-Jones et al, 2016): "(...) es la colaboración entre neurocientíficos, psicólogos y educadorestrabajando a través de los niveles de explicación-que caracteriza a la investigación de la neurociencia educacional" (p. 623) ${ }^{4}$.

Ejemplos de posible interdisciplina entre las ciencias de la educación hallamos en la editorial de Martínez-Montes, Chobert y Besson

\footnotetext{
4 "(...) it is the collaboration of neuroscientists, psychologists, and educators-working across levels of explanation-that characterizes EN research" (Howard-Jones et al, 2016, p. 623)
}

(2016). Los autores señalan trabajos como el de Berteletti y Booth (2015) donde se indagan relaciones entre la información somatosensorial desde los dedos y las habilidades aritméticas y cuyos hallazgos pueden conducir a repensar la utilización del conteo manual en las operaciones con cantidades. Sin embargo, la investigación específica que tome los hallazgos neurobiológicos y combine con la educación es un área propia de la educación, la de la neurociencia educacional. Esta área, a su turno, deberá conversar con la didáctica de las matemáticas en el tema específico de la enseñanza de la aritmética. Otro de los ejemplos que mencionan Martínez-Montes et al (2016) es el trabajo de Danna y Valey (2015) sobre el feedback sensorial en el aprendizaje y la enseñanza de la escritura.

Nuevamente, como dijimos en nuestra observación anterior, será métier de la neurociencia educacional tomar estos resultados e indagarlos desde sus investigaciones y en diálogo con la didáctica de la escritura y quizás otras ciencias de la educación también.

Otro muy buen ejemplo de las intersecciones necesarias en educación puede surgir a partir del trabajo que están realizando investigadores en Bangladesh sobre los efectos de la pobreza en el desarrollo (cfr. Storrs, 2017). Con importantes antecedentes propios y ajenos, el grupo investigadores está conduciendo un estudio de seguimiento de niños desde sus 
primeros días de vida y que actualmente están en edad de la escolaridad primaria. El estudio incluye múltiples variables entre las que se encuentran las provenientes de métodos de neuroimagen y se están analizando cuestiones tales como si estos estudios contienen predictores del desempeño escolar de los niños. Lo que está ofreciendo este estudio a la educación no es una solución práctica para los problemas educacionales sino un ingrediente para trabajar en conjunto la neurociencia educacional con otras ciencias de la educación en función de analizar factores históricos y actuales que pueden influir en el desempeño escolar, a fin de buscar alternativas educacionales. Estas alternativas van más allá de la educación formal, pues puede incluirse la educación no formal y otras instituciones educacionales no sistemáticas y sanitarias a fin de ampliar las acciones de promoción de la salud y prevención primaria y secundaria. Asimismo, estos estudios interdisciplinarios de la neurociencia educacional con ciencias de la educación como política y administración de la educación podrían servir a la toma de decisiones política en función del mejoramiento de las condiciones de vida de todos los habitantes.

\section{Horizontes, desafíos y posibilidades}

Bruer (2016b) señala, sobre la base de su análisis bibliométrico, que la neurociencia educacional se conecta con la investigación psicológico-neurocientífica sobre los mecanismos sinápticos mediante el interés común en el rol de las emociones en el aprendizaje. Esta conexión es nueva y frágil pero el autor supone que se fortalecerá con el tiempo, aunque no está claro cómo influenciará esta línea de investigación el interés central sobre la motivación en el aula de clases visto en la literatura educacional. Otro de los vínculos hallados en el análisis es entre la neurociencia educacional y la investigación sobre memoria de trabajo y cognición, mediante el interés común en la lectura y la dislexia.

Lo que destaca el autor es que la literatura de la neurociencia educacional tiende a ser más metaliteratura que literatura de investigación, esto es, más acerca del concepto de neurociencia educacional y los alcances presentes $y$ futuros que sobre investigaciones concretas de neurociencia educacional (Bruer, 2016a, 2016b).

Sin embargo, si queremos buscar ejemplos de investigaciones que potencialmente puedan proveer insights para la práctica de la enseñanza escolar, podemos enumerar los trabajos citados en el artículo mencionado más arriba de Martínez-Montes et al. (2016).

Una pregunta al respecto es si necesariamente el valor de existencia de la neurociencia educacional está dado por su habilidad de intervenir en las prácticas de enseñanza escolar. Si no es así y pensamos a la 
neurociencia educacional con un valor más teórico la cuestión puede ser diferente. ¿Qué nos puede aportar la neurociencia educacional para educar a lo largo y a lo ancho de la curva neural?, ¿qué aporta al ejercicio de los derechos de todos los ciudadanos?, ¿qué puede aportar para formar en el respeto al otro?, ¿cómo puede colaborar en el proceso de conocerse a sí mismo -un aprendizaje que resulta fundamental para la toma de decisiones que involucra motivación, emociones $y$ resolución de problemas?

\section{Considerando que la} educación, como concepto y forma del hacer humano, es mucho más que la educación formal y que los sujetos de la educación son muchos más que los estudiantes de la educación formal, podemos considerar que la neurociencia educacional puede aportar a la educación miradas amplias que tomen desde la concepción (cfr. por ejemplo un caso de efectos de la experiencia prenatal en Kisilevsky et al, 2003) e incluso desde previamente a la concepción del individuo (cfr. las recomendaciones del cuidado antes de la concepción para la salud de madres e hijos de la Organización Mundial de la Salud, Howson, Kinney y Lawn, 2012) hasta el deceso del individuo.

En el caso de la pregunta sobre qué es la educación como crianza, la neurociencia educacional puede traer a cuenta investigaciones de áreas y disciplinas que conforman las neurociencias que prueban que cuando la crianza (recordemos que la crianza es la primera educación) no es adecuada, el crecimiento del niño (incluyendo peso, altura y circunferencia craneana) es menor al de niños con crianzas adecuadas. La dieta puede $\mathrm{O}$ no haber sido insuficiente, pero un factor clave, junto a la incorporación de nutrientes, es el medioambiente de crianza. De este modo, Johnson y Gunnar (2011) señalan que "La privación psicosocial en cualquier medioambiente de cuidado durante la vida temprana puede verse con tanta preocupación como cualquier enfermedad infantil severamente debilitante" (p. 19) 5 .

Existen periodos de la vida donde la presencia de cierta experiencia vivencial resulta necesaria para el desarrollo del individuo. Esto no significa que los niños deban estar inmersos en un ambiente especialmente diseñado $y$ con estímulos sofisticados. Tampoco implica que el hecho de concebir que estas situaciones de privación puedan ser perjudiciales para el normal desarrollo del individuo signifique que la educación no pueda intervenir de algún modo.

La consideración de la existencia de periodos críticos en el desarrollo requiere de una revisión desde la neurociencia educacional que no conduzca a una

\footnotetext{
5 "Psychosocial deprivation within any caregiving environment during early life must be viewed with as much concern as any severely debilitating childhood disease" (Johnson y Gunnar, 2011, p. 19)
} 
interpretación

equivocada

(generadora de neuromitos).

Greenough, Black y Wallace (1987)

definieron dos procesos que

posiblemente no sean

independientes uno de otro aunque sí distinguibles y que ayudan a la neurociencia educacional a comprender mejor el desarrollo: almacenamiento de la información expectante de la experiencia (experience-expectat information storage) y almacenamiento de la información dependiente de la experiencia (experience-dependent information storage). Mientras que la plasticidad expectante de la experiencia se refiere a cambios en la organización cerebral que ocurren en todos los individuos de una especie y que probablemente suceden dentro de ciertos límites temporales del desarrollo (como los cambios asociados a las experiencias sensoriales), la plasticidad dependiente de la experiencia se refiere a respuestas que varían entre los miembros de una especie y que permiten al individuo adaptarse a su particular medioambiente (tales como cambios asociados al aprendizaje de determinadas habilidades escolares o de la lengua materna que es específica de cada región) (Ansari et al., 2017; Greenough et al., 1987).

Como señala Bruer

cuando se refiere a la interpretación neuromítica de los resultados de las investigaciones sobre ambiente enriquecido y cerebro, la plasticidad expectante de la experiencia no requiere de experiencias específicas en medioambientes específicos, sino que las experiencias necesarias para desarrollar las habilidades sensoriomotoras y de lenguaje fundamentales aparecen en cualquier ambiente normal y esto responde a razones evolutivas y culturales. Evolutivamente hablando, una especie cuyo desarrollo requiriera de medioambientes sofisticados $y$ altamente específicos seguramente no hubiese prosperado como lo ha hecho el Homo sapiens. Por otro lado, las diversas formas culturales de crianza justifican pensar que lo que el cerebro necesita para desarrollarse puede ser provisto de muy distintas maneras.

Lo que hemos aprendido también de los estudios neurobiológicos sobre privación temprana en niños es que es posible una recuperación; por ejemplo, se ha visto que la recuperación en términos de altura es un predictor biológico de la recuperación cognitiva (Johnson y Gunnar, 2011).

La privación emocional y social constituye un factor para el desarrollo cerebral y el aprendizaje. Sin embargo, también se han visto parámetros anormales de desarrollo cerebral en niños en situación extrema de pobreza pero que no carecen de un ambiente familiar, como es el caso del estudio que se está conduciendo en Bangladesh mencionado previamente (Storrs, 2017), donde lo que está en juego es la sanidad y la escasez de nutrientes, en conjunto o no con situaciones 
emocionalmente relevantes como la depresión materna.

De esta forma, no se justifica el uso del conocimiento científico para desarrollar emprendimientos comerciales que supuestamente son los necesarios para el desarrollo del potencial del niño (como la recomendación de ciertos juegos para el cerebro o ciertos jardines de infantes con estímulos sofisticados) pero sí es necesario conocer los efectos que puede tener la privación contextual temprana y cómo puede la educación abordar este problema para lograr, aquí sí, el desarrollo máximo posible del potencial humano más allá de las condiciones desfavorables.

Considerando lo anteriormente dicho, la neurociencia educacional puede ser un elemento importante dentro del curriculum de la formación docente (Ansari et al., 2017) y de la formación de aquellos que se dedican a la educación informal y no formal.

Asimismo, la neurociencia educacional, con la ventaja que le da el acceso a los conocimientos de las neurociencias, puede tener a su cargo la responsabilidad, junto con la política y la administración de la educación, de discutir con conocimiento de causa el presupuesto educacional a nivel político y económico de un país o distrito, donde el modelo popular de acumulación del capital humano learning begets learning (HowardJones, Washbrook, \& Meadows, 2012, p. 19) se muestra simplificador y hasta erróneo para la toma de decisiones de la educación de un país, ya que termina favoreciendo a los sujetos que desde el comienzo han tenido más oportunidades (cfr. por ejemplo, Howard-Jones et al., 2012).

\section{Conclusión}

La neurociencia educacional así entendida puede fomentar la tolerancia, el respeto, la atención a la diversidad $y$ el interés por lo particular, dando lugar a la relevancia de las historias de vida de cada persona. Esta comprensión puede conducir a que diversidad no sea un concepto teórico sino que se refleje en cada ser humano, a que se reivindique el respeto por las distintas generaciones y las potencialidades educativas de cada sujeto en cada etapa y condición de su ciclo vital, a humanizar la educación nominal -la educación de conceptos, no de personas, educación de lo general, no de los particulares-, a revalorizar el respeto por la naturaleza y el entorno, a la valoración de lo distinto y de las otras especies -iqué haríamos sin nuestra flora intestinal y todos los microorganismos que conviven con nosotros!- y a la revisión de la multisensorialidad en situaciones de enseñanza-aprendizaje en cualquier contexto educacional.

Esto no significa que la neurociencia educacional es la nueva pedagogía sino que la neurociencia educacional constituye un campo del 
saber de la educación que puede trabajar en conjunto con el resto de las ciencias de la educación para buscar el desarrollo pleno de las personas en relación armoniosa con su medioambiente de vida.

\section{Bibliografía}

Ansari, D., König, J., Leask, M., \& Tokuhama-Espinosa, T. (2017). Developmental cognitive neuroscience: Implications for teachers' pedagogical knowledge. En: S. Guerriero (Ed.). Pedagogical knowledge and the changing nature of the teaching profession (pp. 195-222). París: OECD Publishing. https://doi.org/10.1787/97892642 70695-11-en.

Bacigalupe, M.A. (2001/2). How neuroscientific studies on memory can help teaching. IATEFL Issues, $164,12$.

Berteletti, I., \& Booth, J.R. (2015). Perceiving fingers in single-digit arithmetic problems. Front Psychol, 6: $226, \quad 1-10$. https://doi.org/10.3389/ fpsyg.2015.00226

Blaze, J., y Roth, T. (2013). Epigenetic mechanisms in learning and memory. WIREs Cogn Sci, 4, 105115.

Bowers, J. (2016a). The practical and principled problems with Educational Neuroscience. Psychol Rev, 123(5), 600-612. https://doi.org/10.1037/rev000002 5
Bowers, J. (2016b). Psychology, not educational neuroscience, is the way forward for improving educational outcomes for all children: Reply to Gabrieli (2016) and Howard-Jones et al. (2016). Psychol Rev, 123(5), 628-635. https://doi.org/10.1037/rev000004 3.

Bruer, J. (1997). Education and the brain: A bridge too far. Educational Researcher, 26(8), 4-16. https://doi.org/10.2307/1176301

Bruer, J. (2016a). Neuroeducación: Un panorama desde el puente. Propuesta Educativa, 46(2), 14-25. Recuperado de: http://www.propuestaeducativa.fla cso.org.ar/archivos/dossier_articul os/102.pdf

Bruer, J. (2016b). Where is educational neuroscience? Educational Neuroscience, 1, 1-12. https://doi.org/10.1177/23776161 15618036

Danna, J., \& Velay, J-L. (2015). Basic and supplementary sensory feedback in handwritting. Front Psychol, 6:169, 1-11. https://doi.org/10.3389/fpsyg.201 5.00169

Dekker, S., Lee, N. C., Howard-Jones, P., \& Jolles, J. (2012). Neuromyths in education: Prevalence and predictors of misconceptions among teachers. Front Psychol, 3, 429, 1-8. https://doi.org/10.3389/fpsyg.201 2.00429 
Dündar, S., \& Gündüz, N. (2016). Misconceptions Regarding the Brain: The Neuromyths of Preservice Teachers. Mind, Brain, and Education, 10(4), 212-232. https://doi.org/10.1111/mbe.1211 9

Gabrieli, J. (2016). The Promise of Educational Neuroscience: Comment on Bowers (2016). Psychol Rev, 123(5), 613-619. https://doi.org/10.1037/rev000003 4

Gardner, H. (1987). The mind's new science: $A$ history of the cognitive revolution. NY, USA: Basic Books.

Greenough, W.T., Black, J.E., y Wallace, C.S. (1987). Experience and Brain Development. Child Development, 58, 539-559.

Howard-Jones, P. (2008). Philosophical Challenges for Researchers at the Interface between Neuroscience and Education. J Philos Educ, 42(3-4), 361-380.

https://doi.org/10.1111/j.1467-

9752.2008.00649.x

Howard-Jones, P., Varma, S., Ansari, D., Butterworth, B., De Smedt, B., Goswami, U. et al. (2016). The principles and practices of educational neuroscience: Comment on Bowers (2016). Psychol Rev, 123(5), 620-627. https://doi.org/10.1037/rev000003 6

Howard-Jones, P., Washbrook, E., \& Meadows, S. (2012). The timing of educational investment: A neuroscientific perspective. Dev Cogn Neurosci, 2(Suppl 1), S18S29.

https://doi.org/10.1016/j.den.2011. 11.002

Howson, C.P., Kinney, M.V., y Lawn, J.E. (Eds.). (2012). Born too soon: the global action report on preterm birth. Geneva: World Health Organization. Recuperado de:

http://www.who.int/pmnch/media /news/2012/201204_borntoosoon -report.pdf

Ibáñez, A., \& García, A.M. (2015). Qué son las neurociencias. Buenos Aires, Argentina: Paidós.

Ito, M. (2001). Cerebellar long-term depression: Characterization, signal transduction, and functional roles. Physiol Rev, 81(3), 11431195.

Johnson D.E., \& Gunnar M.R. (2011). IV. Growth Failure in Institutionalized Children. Monogr Soc Res Child Dev, 76(4), 92-126. https://doi.org/10.1111/j.15405834.2011.00629.x

Kisilevsky B., Hains, S., Lee, K., Xie, X.,

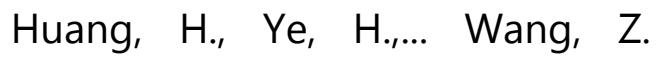
(2003). Effects of experience on fetal voice recognition. Psychol Sci, 14(3), 220-224.

Lipina, S. (2016). Introducción. Actualizaciones en neurociencia educacional. Propuesta Educativa, 46(2), 6-13. Recuperado de: http://www.propuestaeducativa.fla cso.org.ar/archivos/dossier/46.pdf 
Lømo, T. (2003). The discovery of long-term potentiation. Phil Trans $R$ Soc Lond B, 358(1432), 617-620. https://doi.org/10.1098/rstb.2002. 1226.

Magazine de Ciencia. (2016). La síntesis evolutiva moderna (Entrevista al Dr. Jorge V. Crisci). Recuperado de: http://magazinedeciencia.com.ar/l a-sintesis-evolutiva-moderna

Martínez-Montes, E., Chobert, J., \& Besson, M. (2016). Editorial: Neuro-Education and NeuroRehabilitation. Front Psychol, 7: 1427, 1-3. https://doi.org/10. 3389/fpsyg.2016.01427

Mayr, E. (1998). El "por qué": La evolución de los organismos. En: E. Mayr. Así es la biología (pp. 193223). Madrid, España: Debate.

Storrs, C. (2017). How poverty affects the brain. Nature, 547(7662):150152.

https://doi.org/10.1038/547150a 